\title{
Shadowing for Pre-Foundation Doctors-Should the Roles Be Reversed?
}

\author{
Neel Sharma
}

Published online: 29 March 2014

(C) International Association of Medical Science Educators 2014

Dear Editor,

It is a well-known fact that new graduates are amateurs when it comes to their initial days on the ward. This is in my opinion no fault of their own. Despite huge investment by the tax payer, for some reason, medical schools are still getting it wrong. Delivery of theory is one thing, but its application is vastly more important. Unfortunately, change is hard to come by and assessments in current practice still focus on rote learning of hard facts. Even when it comes to the oral components, there seems to be more emphasis on presentation style as compared to what the candidate truly knows and how they may adapt theory to the patient in front of them.

For some time now there has been a requirement for all new starters to undergo pre-foundation shadowing, where they are expected to shadow juniors in their current role, with the supposed expectation of seeing the art of history taking, examination, and procedural skills live in action, not to mention the day to day running of the ward and expectations of the boss.
Educationalists aim to paint a pretty picture of such intervention by choosing to undertake studies focusing on candidate perception of shadowing, either through a post evaluation or pre-/post-medium. We all know that there is likely to be a biased outcome in this regard. After all, which new starter would dare critique a shadowing program when they are already aware of the dangers of whistleblowing way before they even obtain their trust ID cards.

For shadowing to be truly effective, why don't we switch roles? Surely, it would prove beneficial for clinicians to actually see what the difficulties for new comers are, the ward pressures now are certainly more intense than they used to be and any deficiencies both clinical or admin wise would be easily highlighted. Despite seniors setting standards in terms of competencies and assessment, I wonder how much they relate such processes to real life. Or, is it simply a case of the 'back in my day' mantra which seems to hinder bridging the gap between junior and senior.
N. Sharma $(\bowtie)$

Division of Gastroenterology and Hepatology, National University, Hospital, 5 Lower Kent Ridge Rd, Singapore 119074, Singapore

e-mail: drneelsharma@outlook.com 